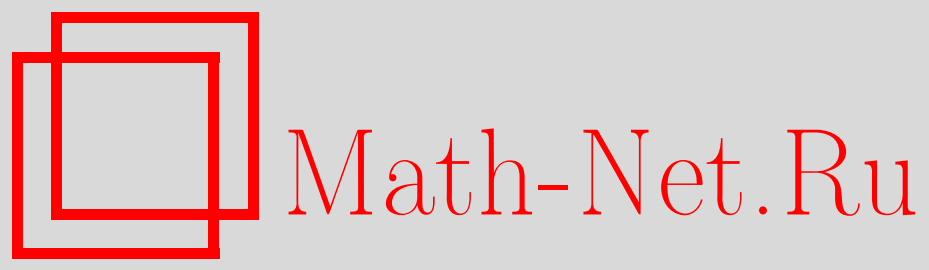

А. В. Стояновский, Деформации алгебр Ли и формулы характера, Функи. анализ и его прил., 1998, том 32, выпуск 1, 84-86

DOI: https://doi.org/10.4213/faa406

Использование Общероссийского математического портала MathNet.Ru подразумевает, что вы прочитали и согласны с пользовательским соглашением

http://www . mathnet.ru/rus/agreement

Параметры загрузки:

IP : 3.89 .185 .249

26 апреля 2023 г., 06:44:39

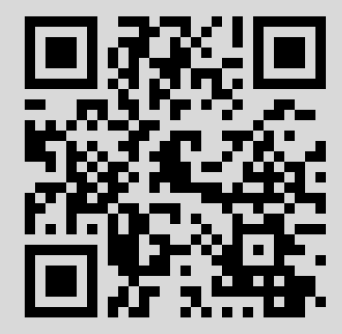


Укажем в заключение статьи [5-7], которые не использовались в настоящей работе, но имеют отношение к ее теме.

\title{
ЛитеРАтУРА
}

1. Кондратьев В. А. ТММО, 16, 209-292 (1967). 2. Назаров С. А., Пламеневский Б. А. Эллиптические задачи в областях с кусочно гладкой границей. Наука, М., 1991. 3. Агранович М. С. Матем. сб., 84(126), № 1, 27-64 (1971). 4. Мазьл В. Г., Пламеневский Б. А. ТММО, 37, 49-93 (1978). 5. Шериф Н. С. А. Вестник МГУ, сер. матем., мех., вып. 3, 7-10 (1980). 6. Мельников И. И. УМН, 37, вып. 1, 149-150 (1982). 7. Eskin G. Commun. Partial Diff. Equat., 17, No. 1, 2, 99-160 (1992).

Санкт-Петербургский государственный университет e-mail: boris.plamenevskij@pobox.spbu.ru
Поступило в редакцию 22 апреля 1997 г.

\section{Деформации алгебр Ли и формулы характера}

\author{
(c) 1998. А. В. Стояновский
}

0. В этой заметке мы докажем серию комбинаторных тождеств

$$
\sum_{m_{1}, \ldots, m_{2 k n} \geqslant 0} \frac{q^{\sum c_{i j} m_{i} m_{j}}}{(q)_{m_{1}} \cdots(q)_{m_{2 k n}}}=\frac{\sum_{\xi \in \check{T}} d((k+n+1) \xi) \cdot q^{\langle\xi, \rho\rangle+(k+n+1)\langle\xi, \xi\rangle / 2}}{(q)_{\infty}^{2 n^{2}+n}}
$$

Здесь использованы следующие обозначения.

В левой части равенства $k \geqslant 0, n \geqslant 1$ - фиксированные целые числа, $(q)_{m}=(1-q)\left(1-q^{2}\right) \cdots\left(1-q^{m}\right)$ и $c_{i j}$ - матричные элементы матрицы $\frac{1}{2} A_{2 n} \otimes \widetilde{B}_{k}^{-1}$ - половины тензорного произведения матрицы Картана $A_{2 n}$ на обратную симметризованную матрицу Картана $B_{k}$,

$$
\left(\widetilde{B}_{k}^{-1}\right)_{i j}=\min (i, j), \quad 1 \leqslant i, j \leqslant k .
$$

В правой части суммирование ведется по решетке $\check{T}$, порожденной длинными корнями алгебры Ли $C_{n}$, т. е. $\mathfrak{s p}(2 n), \rho$ - полусумма положительных корней в $C_{n},\langle$,$\rangle - нормализованная инвариантная билинейная форма,$ $d(\lambda)$ - виртуальная размерность, т. е. полиномиальная функция от $\lambda$, равная размерности неприводимого представления со старшим весом $\lambda$, когда $\lambda$ - доминантный целочисленный вес, и $(q)_{\infty}=\prod_{i=1}^{\infty}\left(1-q^{i}\right)$.

Наши тождества - в некотором смысле родственники известных тождеств Гордона. Напомним, что в работе [1] мы интерпретировали тождества Гордона в терминах теории представлений аффинной алгебры Ли $\widehat{\mathfrak{s l}}(2)$. Новые тождества $(*)$ имеют аналогичную интерпретацию в терминах алгебры Ли $\widehat{\mathfrak{s l}}(2 n+1)$. Тождества для алгебры Ли $\widehat{\mathfrak{s l}}(2 n)$ при $n>1$ остаются пока неизвестными.

1. Сначала о левой части тождеств $(*)$. Если $\mathfrak{a}$ - алгебра Ли, то символом $\hat{\mathfrak{a}}$ будем обозначать соответствующую алгебру токов $\mathfrak{a} \otimes \mathbb{C}((t))$ (центрально 
расширенную, если $\mathfrak{a}$ полупроста). Пусть $\mathbb{V}_{0}$ - вакуумное интегрируемое неприводимое представление алгебры Ли $\widehat{\mathfrak{s l}}(2 n+1)$ на уровне $k, k \in \mathbb{Z}, k \geqslant 0$ (т.е. представление со старшим весом $(0, k))$. Пусть $v_{0} \in \mathbb{V}_{0}-$ вакуумный вектор. Рассмотрим подпространство $W_{0}=U(\hat{\mathfrak{n}}) \cdot v_{0} \subset \mathbb{V}_{0}, \Gamma$ де $\mathfrak{n}$ - верхнетреугольная подалгебра Ли в $\mathfrak{s l}(2 n+1)$, а $U$ - универсальная обертывающая. В $[1, \S 1]$ мы анонсировали формулу характера для подпространства $W_{0}$. Это и есть левая часть тождества $(*)$.

Правая же часть тождества $(*)$ есть не что иное, как формула ВейляКаца [2] для характера вакуумного неприводимого представления алгебры Ли $\widehat{\mathfrak{s p}}(2 n)$ на уровне $k$.

Таким образом, нам осталось доказать следующую теорему:

Теорема 1. Характер вакуумного представления алгебры Ли $\widehat{\mathfrak{s p}}(2 n)$ на уровне $k$ равен характеру подпространства $W_{0}$ в вакуумном представлении алгебры Ли $\widehat{\mathfrak{s l}}(2 n+1)$ на том же уровне.

ЗАМЕЧАНИЕ 1 . В случае $n=1, \mathfrak{s p}(2)=\mathfrak{s l}(2)$, теорема 1 обсуждалась в $[1, \S 4]$.

ДОКАЗАТЕЛЬСТВо ТЕОРЕмы 1. Идея доказательства: и алгебра Ли $\mathfrak{s p}(2 n)$, и алгебра Ли $\mathfrak{n}$ вложены в алгебру Ли $\mathfrak{s l}(2 n+1)$. Мы деформируем подалгебру $\mathfrak{s p}(2 n)$ в подалгебру $\mathfrak{n}$ и докажем, что характер соответствуюшего подпространства в вакуумном представлении $\mathbb{V}_{0}$ алгебры Ли $\widehat{\mathfrak{s l}}(2 n+1)$ не меняется при деформации.

2. Деформация подалгебры Ли $\mathfrak{s p}(2 n) \subset \mathfrak{s l}(2 n+1)$ в верхнетреугольную подалгебру Ли $\mathfrak{n}$. Эта конструкция принадлежит Б. Л. Фейгину.

Пусть $e_{0}, e_{1}, \ldots, e_{2 n}$ - базис в стандартном $(2 n+1)$-мерном векторном пространстве $V$. Рассмотрим подалгебру Ли $\mathfrak{g}_{\varepsilon} \subset \mathfrak{s l}(2 n+1)$, аннулирующую вектор $e_{0}$ и аннулирующую кососимметричную билинейную форму

$$
\beta_{\varepsilon}=e_{0} \wedge e_{1}+\varepsilon e_{1} \wedge e_{2}+\varepsilon^{2} e_{2} \wedge e_{3}+\cdots+\varepsilon^{2 n-1} e_{2 n-1} \wedge e_{2 n} .
$$

Мы утверждаем, что при $\varepsilon \neq 0$ эта подалгебра сопряжена $\mathbf{c} \mathfrak{s p}(2 n) \subset \mathfrak{s l}(2 n+1)$. Это очевидно, если учесть, что $\beta_{\varepsilon}^{n} \wedge e_{0} \neq 0 \in \wedge^{2 n+1} V$. С другой стороны, посмотрим, во что вырождается эта подалгебра при $\varepsilon \rightarrow 0$. Вот список инвариантов нашей алгебры Ли $\mathfrak{g}_{\varepsilon}$ во внешней алгебре $\wedge^{*} V$ :

$$
\begin{aligned}
& \text { B } \Lambda^{1} V \quad e_{0} \text {, } \\
& \text { в } \Lambda^{2} V \quad \beta_{\varepsilon}=e_{0} \wedge e_{1}+o(1), \\
& \text { в } \Lambda^{3} V \quad e_{0} \wedge \beta_{\varepsilon}=\varepsilon e_{0} \wedge e_{1} \wedge e_{2}+o(\varepsilon) \text {, } \\
& \text { в } \Lambda^{2 i-1} V \quad e_{0} \wedge \beta_{\varepsilon}^{i-1}=\varepsilon^{(i-1)^{2}} e_{0} \wedge e_{1} \wedge \cdots \wedge e_{2 i-2}+o\left(\varepsilon^{(i-1)^{2}}\right) \text {, } \\
& \text { в } \Lambda^{2 i} V \quad \beta_{\varepsilon}^{i}=\varepsilon^{i^{2}-i} e_{0} \wedge e_{1} \wedge \cdots \wedge e_{2 i-1}+o\left(\varepsilon^{i^{2}-i}\right) \text {, }
\end{aligned}
$$

в $\Lambda^{2 n+1} V \quad e_{0} \wedge \beta_{\varepsilon}^{n}=\varepsilon^{n^{2}} e_{0} \wedge e_{1} \wedge e_{2} \wedge \cdots \wedge e_{2 n}$. 
Отсюда видно, что при $\varepsilon \rightarrow 0$ наша подалгебра стремится (в смысле топологии грассманиана) к верхнетреугольной подалгебре $\mathfrak{n} \subset \mathfrak{s l}(2 n+1)$.

3. Рассмотрим теперь семейство подпространств $W_{\varepsilon} \stackrel{\text { def }}{=} U\left(\hat{\mathfrak{g}}_{\varepsilon}\right) \cdot v_{0} \subset \mathbb{V}_{0}$. При $\varepsilon \neq 0$ подпространство $W_{\varepsilon}$ есть вакуумное представление алгебры Ли $\hat{\mathfrak{g}}_{\varepsilon} \cong \widehat{\mathfrak{s p}}(2 n)$, а при $\varepsilon=0$ подпространство $W_{0}$ получается действием универсальной обертывающей $U(\hat{\mathfrak{n}})$ на вакуумный вектор $v_{0}$. Докажем, что характеры подпространств $W_{\varepsilon}$ и $W_{0}$ равны, и тем самым теорема будет доказана.

Обозначим через $E_{i j}$ стандартный матричный элемент алгебры Ли $\mathfrak{s l}(2 n+1)$. Пусть $J_{\varepsilon}-$ левый идеал алгебры $U\left(\hat{\mathfrak{g}}_{\varepsilon}\right)$, аннулирующий вектор $v_{0}$. Это фильтрованное пространство, причем присоединенные факторы фильтрации градуированы и их градуировочные компоненты конечномерны. Из линейной алгебры ясно, что в специальной точке $\varepsilon=0$ идеал $J_{\varepsilon}$ может только подскочить (а характер пространства $W_{\varepsilon} \simeq U\left(\hat{\mathfrak{g}}_{\varepsilon}\right) / J_{\varepsilon}$, наоборот, уменьшиться). Нам осталось доказать, что идеал не подскочит, т. е. все элементы идеала $J_{0}$ деформируются в некоторые элементы идеала $J_{\varepsilon}$. В работе $[1, \S 1]$ мы анонсировали, что образующие левого идеала $J_{0}$ - это, вопервых, «положительная часть» $\mathfrak{n} \otimes \mathbb{C}[[t]]$ алгебры $\hat{\mathfrak{n}}$ и, во-вторых, двусторонние образующие, а именно, фурье-компоненты выражений $E_{i+1, i}(z)^{k+1}$, где $E_{i+1, i}(z)=\sum_{j \in \mathbb{Z}}\left(E_{i+1, i} \otimes t^{j}\right) z^{-j-1}$ — ток матричного элемента $E_{i+1, i} \in \mathfrak{n}$.

Рассмотрим теперь идеал $J_{\varepsilon}$. Он, прежде всего, очевидно, содержит положительную часть $\mathfrak{g}_{\varepsilon} \otimes \mathbb{C}[[t]]$. Рассмотрим, далее, элементы

$$
E_{i+1, i}^{\varepsilon}=E_{i+1, i}-\varepsilon E_{i+1, i+2} \in \mathfrak{g}_{\varepsilon} .
$$

Эти элементы нильпотентны, а следовательно, для них выполняется соотношение $E_{i+1, i}^{\varepsilon}(z)^{k+1} \in J_{\varepsilon}$ (например, по теореме Джекобсона-Морозова и потому, что эти соотношения выполняются для $\widehat{\mathfrak{l l}}(2))$. Таким образом, мы видим, что образующие (а значит, и все элементы) идеала $J_{0}$ деформируются в элементы из $J_{\varepsilon}$, что и требовалось доказать.

ЗАмечАниЕ 2. Как указал нам Д. А. Панов, существует более очевидный пример деформации простой подалгебры Ли в нильпотентную: подалгебра Ли $\mathfrak{s o}(n) \subset \mathfrak{s l}(n)$ вырождается в верхнетреугольную подалгебру Ли после сопряжения матрицей $\operatorname{diag}\left(1, \varepsilon, \varepsilon^{2}, \ldots, \varepsilon^{n}\right)$ при $\varepsilon \rightarrow 0$. Однако в этом случае утверждение, аналогичное теореме 1, неверно: даже показатели роста у характеров различны.

Я хотел бы поблагодарить своего учителя Б. Л. Фейгина за постановку задачи, помощь при решении и теплую поддержку при работе над заметкой.

\section{ЛИТЕРАТУРА}

1. Стояновский А. В., Фейаин Б. Л. Функц. анализ и его прил., 28, вып. 1, 68-90 (1994). 2. Кач В. Бесконечномерные алгебры Ли. Мир, М., 1993.

Независимый московский университет Институт теоретической физики

Поступило в редакцию им. Л. Д. Ландау РАН

13 мая 1997 г. 